\title{
Physical health risks during simulation-based COVID-19 pandemic readiness training
}

\author{
Michelle Chiu, MD, FRCPC · Simone Crooks, MD, FRCPC · Amy B. Fraser, MD, \\ MSc, FRCPC, MHPE · Purnima Rao, MD, FRCPC · Sylvain Boet, MD, PhD
}

Received: 3 June 2020/Revised: 10 June 2020/Accepted: 10 June 2020/Published online: 25 June 2020

(C) Canadian Anesthesiologists' Society 2020

\section{To the Editor,}

Simulation is an effective modality for team training under realistic conditions; it is commonly used to practice new, complex, or uncommon procedures without incurring risks to patients. ${ }^{1}$ Simulation can be invaluable when creating clinical or educational tools that require real-time practice and team coordination, particularly when a novel, rapidly evolving healthcare crisis mandates adaptations in

M. Chiu, MD, FRCPC

Department of Anesthesiology and Pain Medicine, University of Ottawa, Civic Campus, 1053 Carling Ave, B3, Ottawa, ON K1Y 4E9, Canada

Department of Innovation in Medical Education, University of Ottawa, Ottawa, Canada

S. Crooks, MD, FRCPC $\cdot$ P. Rao, MD, FRCPC

Department of Anesthesiology and Pain Medicine, University of Ottawa, General Campus, 501 Smyth Rd, Critical Care Wing

1401, Ottawa, ON K1H 8L6, Canada

\section{A. B. Fraser, MD, MSc, FRCPC, MHPE}

Department of Anesthesiology and Pain Medicine, University of Ottawa, Civic Campus, 1053 Carling Ave, B3, Ottawa, ON K1Y 4E9, Canada

\author{
S. Boet, MD, PhD ( $\square)$ \\ Department of Anesthesiology and Pain Medicine, University of \\ Ottawa, General Campus, 501 Smyth Rd, Critical Care Wing \\ 1401, Ottawa, ON K1H 8L6, Canada \\ e-mail: sboet@toh.ca
}

Department of Innovation in Medical Education, University of Ottawa, Ottawa, Canada

Office of Francophone Affairs, Faculty of Medicine, University of Ottawa, Ottawa, Canada

Faculty of Education, University of Ottawa, Ottawa, ON, Canada both the work environment and team functioning. The usual risks to healthcare workers (HCWs) during training via simulation are most often limited to psychologic safety considerations (e.g., stress). ${ }^{2,3} \mathrm{We}$ describe a case of physical health risk resulting from simulation-based HCW training to prepare for the coronavirus disease (COVID-19) pandemic.

In anticipation of the increasing likelihood of caring for COVID-19 patients in the perioperative environment, our University's Department of Anesthesiology and Pain Medicine Simulation Education Team (ASET) rapidly mobilized to deliver simulation training. This included personal protective equipment (PPE) training, and two in situ interprofessional simulation scenarios to perioperative staff, including that of a stable COVID-19 patient presenting to the operating room (OR) for emergency surgery and an unstable COVID-19 patient requiring urgent intubation in a remote emergency department isolation room. One hundred and fifty staff were trained in PPE donning and doffing procedures over two days (16-17 March 2020). Two hundred and thirtynine staff participated in the simulations over three days (18-20 March 2020): 99 anesthesiologists (staff, residents, fellows), 34 surgeons (staff, residents, fellows), and 106 additional HCWs (nurses, anesthesia assistants, and OR attendants). The training sessions were extremely well received, generating very positive feedback from participants and hospital leaders.

Nevertheless, four days after the simulation training, our simulation educators were informed by the hospital occupational health and safety administration (OHSA) office that one of the simulation participants was being tested for severe acute respiratory syndrome coronavirus 2 (SARS-CoV-2). All HCWs who came into contact with that individual were risk-stratified by the OHSA office 
Table 1 Suggestions on mitigating the risks of virus transmission during COVID-19 pandemic training via simulation

Instructing participants to decline participation if they display any symptoms suggestive of COVID-19

Scheduling small groups to facilitate physical distancing

Assigning an individual to enforce physical distancing measures

Using dated sign-in sheets in each scenario to track participants who drop-in/out of the sessions

Organizations and governments must stock adequate PPE to minimize temptation of using and reusing supplies during training, and to permit educators to "waste" PPE during training sessions. In our case, reuse of PPE between participants was likely not a large factor, given that face shields were sanitized between uses and individuals used their own masks (i.e., expired N95) and disposable gloves

Filming scenario videos showing expert performance to facilitate education, future review, or for use if face-to-face staff training is impossible, and distribute with key debriefing points

Advocating for ongoing pandemic preparatory training and proactively delivering simulation-based training as it is difficult to predict when these circumstances occur. Healthcare organizations could collaborate with Infection Prevention and Control offices to set a threshold at which to institute simulation-based training. Healthcare organizations and educators must learn from experience. We saw the approaching surge of COVID-19 patients and should have initiated simulation training earlier, probably as soon as the World Health Organization declared a Public Health Emergency of International Concern on 30 January 2020.*

*World Health Organization. Rolling updates on coronavirus disease (COVID-19). Geneva, Switzerland: April 2020. Available from URL: https://www.who.int/emergencies/diseases/novel-coronavirus-2019/events-as-they-happen (accessed June 2020).

COVID-19 = coronavirus disease; PPE = personal protective equipment.

according to the time spent in close proximity and type of interaction with the index participant, and were given instructions on how to proceed (e.g., self-monitor, selfisolate). Because of Personal Health Information Protection Act legislation, no details on the participant's symptomatology, reason for testing, or outcomes of contact tracing were disclosed. Subsequent to this event, the ASET cancelled all subsequent simulation sessions as it was felt that the risks of contracting and transmitting SARS-CoV-2 amongst HCWs outweighed the educational benefits. Subsequent education was then provided by online dissemination of airway management guidelines for the COVID-19 patient, new surgical safety checklist content, and $360^{\circ}$ spherical virtual reality content videos of both simulation scenarios. ${ }^{\mathrm{A}, \mathrm{B}}$

Risks to simulation participants have rarely been reported and have generally been overlooked. This is a report of non-equipment-related physical health risk to simulation participants. Several factors may have contributed to this risk. Although physical distancing policies were active at our institution and educators advised physical distancing in the pre-scenario brief, this was difficult to accomplish in practice. As educators were fully engaged in running and debriefing simulation scenarios, they were unable to enforce physical

\footnotetext{
A Rao P, Chiu M, Fraser AB. Case 3: COVID-19 Patient Requiring Surgery. The Ottawa Hospital, University of Ottawa, and University of Colorado School of Medicine, April 2020. Available from URL: https://crud.spaces.wondavr.com/api/courses/10fcd570-6c9a-11ea86c9-f71bbff357bd/room (accessed June 2020).

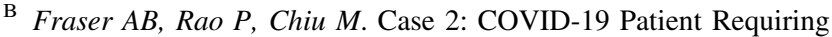
Anesthesia. The Ottawa Hospital, University of Ottawa, and University of Colorado School of Medicine, April 2020. Available from URL: https://spaces.wondavr.com/embed/?course=37443aa06c60-11ea-86c9-f71bbff357bd (accessed June 2020).
}

distancing measures. Group sizes were larger than desired (up to 25 people) because of the urgency to rapidly train all staff. Participant "buy-in" (or "suspension of disbelief"), the limited size of the isolation room used, and difficulty hearing the masked HCWs may also have brought participants in closer proximity. Table 1 provides some suggestion to mitigate the risks identified above.

We hope that this report will be useful for educators planning to use simulation as an educational modality in preparing HCWs for the COVID-19 pandemic and beyond. Modifications should be considered to usual best practices in simulation-based education when training for $\mathrm{HCWs}$ pandemic readiness. In additional to psychologic safety, it is important to consider the potential physical health risks to HCWs with respect to equipment used, and balance the risk of viral transmission between participants with the benefits of experiential learning.

Acknowledgement The authors thank Dr. Donald Miller for his comments on an earlier version of this manuscript.

Disclosures None.

Funding statement All authors were supported by The Ottawa Hospital Anesthesia Alternate Funds Association.

Editorial responsibility This submission was handled by Dr. Hilary P. Grocott, Editor-in-Chief, Canadian Journal of Anesthesia.

\section{References}

1. Cook DA, Hatala $R$, Brydges $R$, et al. Technology-enhanced simulation for health professions education: a systemic review and meta-analysis. JAMA 2011; 306: 978-88. 
2. Clarke S, Horeczko T, Cotton D, Bair A. Heart rate, anxiety and performance of residents during a simulated critical clinical encounter: a pilot study. BMC Med Educ 2014. DOI: https://doi. org/10.1186/1472-6920-14-153.

3. LeBlanc VR. The effects of acute stress on performance: implications for health professions education. Acad Med 2009; 84(10 Suppl): S25-33.
Publisher's Note Springer Nature remains neutral with regard to jurisdictional claims in published maps and institutional affiliations. 